\section{variety, complexity hard to fathom}

\author{
napira.jrc.ec.europa.eu \\ www.goodnanoguide.org \\ www.nano.gov
}

C onfused by contradictory scientific reports about the hazards deriving from nanomaterials, and about how these hazards are measured? You're not alone.

Combined with different definitions and a lack of validated characterization criteria and methods, the complexity and large variety of nanomaterials make general statements about their potential health risks and safety issues meaningless.

Assessing the risks posed by nanomaterials properly means investing time and money on toxicity and other studies for each type of nanoparticle.

While the precise parameters for defining nanomaterials are debated internationally, the National Nanotechnology Initiative (NNI) and its member agencies in the United States define engineered nanomaterials as "materials that have been purposefully manufactured, synthesized, or manipulated to have a size with at least one dimension in the range of approximately 1 to 100 nanometers and that exhibit unique properties determined by their size." US regulatory agencies add mission-specific context to this definition to meet their regulatory responsibilities.

The Joint Research Council (JRC) of the European Union (EU) says there is "little dispute that nanomaterials are an interesting class of materials because some of their properties are different from [read 'better than'] the properties of equivalent bulk materials."

But, the JRC adds, "These distinguishing properties are not the same for each nanomaterial. Most nanomaterial definitions are therefore not based on nano-specific functional properties but often solely on size as the structural feature that is the most common denominator for all kinds of nanomaterials."
In 2011, the EU adopted its definition: "Nanomaterial' means a natural, incidental or manufactured material containing particles, in an unbound state or as an aggregate or as an agglomerate and where, for $50 \%$ or more of the particles in the number size distribution, one or more external dimensions is in the size range $1 \mathrm{~nm}-100 \mathrm{~nm}$."

As this definition focuses on particulate matter and the external size of the nanoparticles that make up the nanomaterials, it provides "an appropriate measurement system that distinguishes nanomaterials from other materials," the JRC says.

Definitions apart, handling guidelines do exist. The US National Institute of Occupational Health and Safety (NIOSH) has issued strategies and recommendations for safe handling of nanomaterials in the workplace, while the US Occupational Safety and Health Administration (OSHA) has issued general industry standards for handling nanomaterials in industrial settings.

NIOSH and OSHA helped develop the GoodNanoGuide to enable experts to exchange ideas on how best to handle nanomaterials in an occupational setting.

Furthermore, the US Environmental Protection Agency (EPA) has specific guidelines, under the Federal Insecticide, Fungicide, and Rodenticide Act (FIFRA), and the Toxic Substances Control Act (TSCA), requiring approvals for nanomaterials in research and pilot production.

An EU directive to improve occupational safety and health obligates employers to protect their workers, including those who deal with nanomaterials in research facilities and pilot production.

Europe also has a number of health and safety guidelines-embodying a responsible and precautionary approach-for handling nanomaterials in production plants, industry, and research laboratories. In fact, new French legislation ("Grenelle 2") requires registration of nanomaterials synthesized and used in research beginning January 2013.

Kenneth A. Dawson, director of the Center for BioNano Interactions (CBNI) at University College Dublin, Ireland, says most research institutions and well-structured companies in the United States and Europe have policies about nanomaterial safety. There have also been good initiatives in Asia, but information from other regions is less clear.

Dawson says, however, that it is unclear whether these intra-institutional policies conform well to each other, and whether they reflect the different levels of experience and expertise of the institutions.

"I believe that there are some discussions within EU programs now to survey this situation more fully," he says. "This might make a very good US-EU collaboration."

Using nanomaterials in research and pilot plant production needs precautionary principles developed through case-by-case assessments, says Keld A. Jensen, a senior researcher at Denmark's National Research Center for the Working Environment.

"Workplace regulation requires that exposure is kept as low as reasonably possible," says Jensen, who researches exposure risks during production and handling of nanoparticles. "This is often easy in small-scale production by, e.g., encapsulation, but may become increasingly difficult with up-scaling until production becomes larger with sufficient earnings."

He adds, "Occupational exposure limits have not been revised and we still lack significant documentation and knowledge to change these limits in the traditional way. This requires testing and, usually, international consensus."

Günter Oberdörster, a professor in the Department of Environmental Medicine at the University of Rochester Medical Center, Rochester, N.Y., researches the toxicology of airborne environmental 


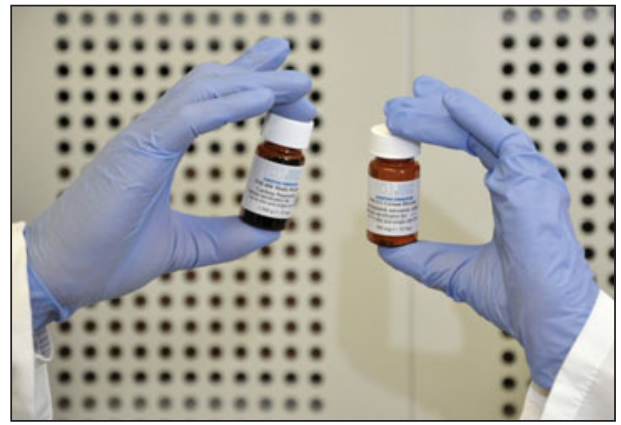

Vials with representative nanomaterials in the JRCIspra repository. (C) EU, 2012.

and occupational particles and nanoparticles. He does not see differing definitions as a major problem for preparing regulations or standards.

"But," he says, "there must be validated methods for characterizing nanomaterials."

Noting that NIOSH has also issued recommendations for exposure levels, Oberdörster asks, "How do you correlate studies with different materials or material types in different situations or contexts? How do you relate these to exposure levels? How do you rank an unknown material? Which criteria should be used? And how should they be determined?"

"In the absence of a more developed science," says Dawson, "and except for specific examples that are now identified, we do not yet know if there are particular nanomaterial characteristics that could pose a threat."
Reference materials are emerging, though many of the early ones are oriented toward size measurements, Dawson says.

"The first reference materials enabling greater emphasis on standard positive and negative controls will be announced soon by the European Infrastructure for Nanosafety, Qnano (http:// www.qnano-ri.eu)," he adds.

Jensen says there is ample evidence of increased hazards associated with many types of nanomaterials.

"The complexity increases with the complexity of the material," he says. "It is not only a simple change in scale with the associated change in properties and ability to reach other biological compartments than their bulk analog materials. Engineering at the particle level has also changed. Size, morphology, deliberate impurities such as catalysts, layers of inorganic and organic coatings, for example, are factors making nanomaterials unique for their intended use. But they potentially also change the toxicity of the material so it is no longer comparable to the bulk analog_if such an analog exists."

The NNI notes that nanomaterial environmental health and safety research is further complicated by the interaction of the materials' high reactivity surface with the microenvironment.

The JRC says more research is needed, including the development of methods tailored to assessing nanomaterials to get a better understanding of toxicity mechanisms. Moreover, data relevant for regulatory risk assessment (e.g., on potential exposure) are still scarce for many materials. More research is necessary just to keep pace with new developments.

According to Eric Gaffet, deputy director of the Institut Jean Lamour Materials Science Institute at the University of Lorraine in France, the toxicity and ecotoxicity of nanomaterials depend on at least eight major parameters.

"You can easily realize the variety of combinations you can get for a given chemically identical nanoparticle," he says. "More than 50 years may be needed to get information on the nanoparticles on the market, at a cost of $€ 3-5$ million Euros [USD\$3.8-6.4 million] per nanoparticle to be tested."

But, Gaffet says, "Instead of considering toxicity and ecotoxicity after new properties have been researched and developed, some people propose considering the toxicity and ecotoxicity as equally important parameters during research and development."

This is the "safer by design" approach to product safety, Gaffet says. "It means developing processes confining nanoparticles, or not producing isolated nanoparticles, and also confining the chemistry of nanoparticles and stabilizing the eight parameters in order to decrease the interaction of a nanoparticle with its biological environment or to decrease its toxicity."

Michael de Laine
India launches fellowship to combine academic

research with industry needs

$\mathbf{L}$ ast November, Jaipal Reddy, India's nnew Minister for Science \& Technology and Earth Science, launched the Prime Minister's Fellowship Scheme for Doctoral Research, being implemented jointly by the Science \& Engineering Research Board and the Confederation of Indian Industry. With this initiative, 100 fellowships are to be awarded annually to research students in the fields of science, technology, engineering, agricul- ture, and medicine. The students are to partner with industry in order to pursue research that fulfills industrial needs. Speaking at a conference in New Delhi, Reddy said that "a seed is being sown today for a transformational change in the way research and development is being promoted in this country."

"The scheme," he said, "offers now a financial incentive for those who are able to align their research interests and engage their creative potentials to solving problems which are seeking solutions rather than to create solutions which would solve no one's problems." While lauding the recent $13-15 \%$ annual growth in research publications from Indian institutions, Reddy said that "the challenge is in the conversion of such knowledge into products of commercial value to the industrial users."

Reddy said that within the next five years, he aims to bring India to the fifth position in Global Science and Technology from the current twelfth position. $\square$ 